\title{
Novos registros de Trachelomonas Ehr. emend. Defl. (Euglenophyceae) para o Estado do Rio Grande do Sul e Brasil
}

\author{
Sandra Maria Alves-da-Silva ${ }^{1,2}$, Karine Corrêa Escobar ${ }^{1}$ e Viviane Berwanger Juliano ${ }^{1}$
}

Recebido: 4.12.2014; aceito: 10.06.2015

\begin{abstract}
New records of Trachelomonas Ehr. emend. Defl. (Euglenophyceae) for the Rio Grande do Sul State and Brazil). The aim of this study was the taxonomic analysis of microalgae from the genus Trachelomonas (Euglenophyceae) in the Environmental Protection Area (APA) of the Ibirapuitã River, located on the southwest border of Rio Grande do Sul State. We sampled 10 stations in lotic and lentic environments, between 2011 and 2013. The 34 samples, resulting from sampling, were collected with a plankton net $(30 \mu \mathrm{m})$, and preserved with $4 \%$ formalin. Nine taxa are new records for Rio Grande do Sul State, found in only three of the 10 stations sampled. Among these, Trachelomonas bernardii Wolosz. $\mathrm{f}$. major Defl., T. caudata Defl. f. pseudocaudata (Defl.) Pop., T. hirta Cunha var. hirta, and T. torosa (Bal.) Tell \& Conf. are first reports for the country.
\end{abstract}

Keywords: Loricate Euglenophyceae, southwest of Rio Grande do Sul State, southern Brazil

RESUMO - (Novos registros de Trachelomonas Ehr. emend. Defl. (Euglenophyceae) para o Estado do Rio Grande do Sul e Brasil). O trabalho objetivou o estudo taxonômico de microalgas do gênero Trachelomonas (Euglenophyceae) na Área de Proteção Ambiental (APA) do rio Ibirapuitã, localizada na fronteira sudoeste do Estado do Rio Grande do Sul. Foram amostradas 10 estações de coleta em ambientes lóticos e lênticos, no período de 2011 a 2013. As 34 amostras resultantes de quatro coletas foram obtidas com rede de plâncton com abertura de malha de $30 \mu \mathrm{m}$, e conservadas com formol a $4 \%$. São apresentados os nove táxons que são novos registros para o Estado do Rio Grande do Sul, encontrados em somente três estações de coleta das 10 amostradas. Dentre esses, distinguem-se ainda por serem citados pela primeira vez para o Brasil: Trachelomonas bernardii Wolosz. f. major Defl., T. caudata Defl. f. pseudocaudata (Defl.) Pop., T. hirta Cunha var. hirta e T. torosa (Bal.) Tell \& Conf.

Palavras-chave: Euglenophyceae loricada, sudoeste do Estado do Rio Grande do Sul, sul do Brasil

\section{Introdução}

Euglenophyceae é uma classe de microalgas flageladas, pigmentadas ou incolores, composta de organismos unicelulares, com exceção do gênero Colacium Ehr. que é colonial. Dessa classe foi estudado o gênero Trachelomonas Ehr. emend. Defl. que apresenta o maior número de espécies dentro da divisão, tendo Guiry \& Guiry (2014) mencionado 325 espécies taxonomicamente aceitas. São organismos unicelulares, fototróficos, cuja célula é semelhante à Euglena, sendo rodeado por uma carapaça denominada de lórica, que pode ser esférica, semi-esférica elipsóide, oblonga, fusiforme ou campanulada (Bicudo \& Menezes 2006). Podendo ser lisa, pontuada, escrobiculada, espinhosa, papilada, com depressões, estriada, granulosa ou tuberculada (Conforti \& Tell 1986). A parede mucilaginosa da lórica varia de amarelada a castanha-escura (Bicudo $\&$ Menezes 2006), dependendo da sua genética e da disponibilidade primordialmente de ferro e manganês no ambiente no qual vivem.

Esse trabalho foi desenvolvido na Área de Proteção Ambiental (APA) do rio Ibirapuitã, uma Unidade de Conservação Federal, localizada no sudoeste do Estado do Rio Grande do Sul, no Bioma Pampa. É um dos resultados do estudo taxonômico e ecológico de algas dentro do programa de Pesquisas Ecológicas de Longa Duração (PELD), sítio 25. Devido à alta diversidade de Euglenophyceae

\footnotetext{
1. Museu de Ciências Naturais da Fundação Zoobotânica do Rio Grande do Sul, Seção de Botânica de Criptógamas. Caixa Postal 1188, 90001-970 Porto Alegre, RS, Brasil

2. Autor para correspondência: sandra-silva@fzb.rs.gov.br
} 
registrada na APA, os resultados serão divididos em várias publicações. $\mathrm{O}$ primeiro trabalho abrangeu os gêneros: Cryptoglena, Monomorphina e Phacus, sendo esta a segunda publicação abordando as novas citações de Trachelomonas para o Estado e/ou Brasil.

Trachelomonas é um gênero cosmopolita ocorrendo principalmente em água doce. Predominantemente em ambientes mesotróficos a eutróficos (Sládecék 1973, Alves-da-Silva \& Ávila, 1995, Wolowski 1998, Giani et al. 1999, Ferreira \& Menezes 2000, Danilov \& Ekelund 2001, Kim \& Boo 2001, Perez 2002, Conforti et al. 2005, Tucci et al. 2006, Alves-da-Silva \& Schüler-da Silva 2007, Alves-da-Silva et al. 2007, Solorzano et al. 2011, Duangjan et al. 2012).

No Estado do Rio Grande do Sul é facilmente encontrado como constituinte da ficoflórula, existindo atualmente o registro de 103 táxons de Trachelomonas em nível de espécie e infraespécie no Estado (Rosa et al. 1987, Alves-da-Silva 1988, Franceschini 1992, Alves-da-Silva \& Laitano 1994, Alves-da-Silva \& Ávila, 1995, Alves-da-Silva \& Schüler-da-Silva 2007, Alves-da-Silva et al. 2007, Alves-da-Silva et al. 2008, Domingues \& Torgan 2011, Alves-da-Silva \& Bicudo 2013).

Trachelomonas foi descrita por Ehrenberg em 1833. Stein (1878), Lemmermann (1913), Playfair (1915) e Conrad (1916) utilizaram apenas características morfológicas em seus estudos. Mais tarde, Deflandre (1926) apresentou, em sua monografia, além da morfologia a descrição de cloroplastos e pirenóides para algumas espécies. Pringsheim (1953), trabalhando com culturas, verificou que a ornamentação da lórica pode variar em resposta às mudanças ambientais. Esse mesmo autor sugeriu que os agrupamentos naturais de táxons dentro do gênero devam considerar ainda, os cloroplastos e a estrutura dos pirenóides, como ocorre no gênero Euglena (Pringsheim, 1956). Apesar da recomendação desse autor, a taxonomia do gênero continuou alicerçada na morfologia da lórica (HuberPestalozzi 1955, Popova 1966, Bourrelly 1970, Starmach 1983, Tell \& Conforti 1986, Shi et al. 1999). Com exceção de alguns autores como Middelhoek (1948, 1951), Rino (1972), Philipose (1988), Rino \& Pereira (1988, 1989-1990) e Kim et al. (2000) que consideram as características protoplasmáticas nas suas identificações do gênero. No Brasil já foram publicados vários trabalhos que consideraram a morfologia da lórica, e alguns apresentaram também, características do protoplasto (Cunha 1913, 1914, Cardoso 1982, Menezes \& Fernandes 1987, Menezes 1991, Xavier 1993, 1994, 1996, Jati \&
Train 1994, Alves-da-Silva \& Ávila 1995, Menezes et al. 1995, Kepeller et al. 2002, Alves-da-Silva \& Schüler-da-Silva 2007, Alves-da-Silva \& Tamanaha 2008, Alves-da-Silva et al. 2008, Araújo et al. 2012, Alves-da-Silva \& Bicudo 2013, Alves-da-Silva et al. 2013). Ao longo do tempo os estudos abrangendo a morfologia da lórica acompanhada de estruturas protoplasmáticas revelaram-se complementares para uma boa identificação de espécies e infraespécies deste gênero.

Três trabalhos resumem os estudos taxonômicos envolvendo comunidades de microalgas aquáticas na fronteira sudoeste do Estado do Rio Grande do Sul: Relatório Brasil das Águas (2007), Matzubara et al. (2008) e Alves-da-Silva \& Klein (2015) abrangendo os gêneros Cryptoglena, Monomorphina e Phacus da APA. Não havendo nenhum registro do gênero Trachelomonas na área estudada.

Do levantamento taxonômico do gênero Trachelomonas na APA foram registrados alguns táxons que são novas citações para o Estado e Brasil. Esta publicação tem como objetivo descrever e ilustrar esses novos registros do gênero, além de contribuir para ampliação do conhecimento da distribuição geográfica dos mesmos no Estado do Rio Grande do Sul e no Brasil.

\section{Material e métodos}

A APA abriga parte da bacia Hidrográfica do rio Ibirapuitã. É dividida longitudinalmente por esse rio que nasce no oeste da cidade de Livramento, percorre por $100 \mathrm{~km}$ a APA no sentido sul-norte (figura 1), indo desaguar em Alegrete, no rio Ibicuí que é afluente da bacia do Uruguai. O rio Ibirapuitã caracteriza-se pela ocorrência predominante de rochas vulcânicas basálticas, sendo parte do leito rochoso e parte arenoso. Em época de chuva alaga grandes áreas e recebe carga orgânica oriunda predominantemente da agricultura (arroz irrigado) e pecuária (bovinos e ovinos).

O clima da região é subtropical, temperado quente (Conti \& Furlan 2003).

Foram realizadas amostragens com rede de plâncton com abertura de malha de $30 \mu \mathrm{m}$, em 10 estações (E1 a E10) na Área de Proteção Ambiental (APA) do rio Ibirapuita (figura 1). As amostras foram subdivididas em duas partes: uma parte foi fixada em campo com formol a $4 \%$, e parte foi mantida viva para observação de características morfológicas 


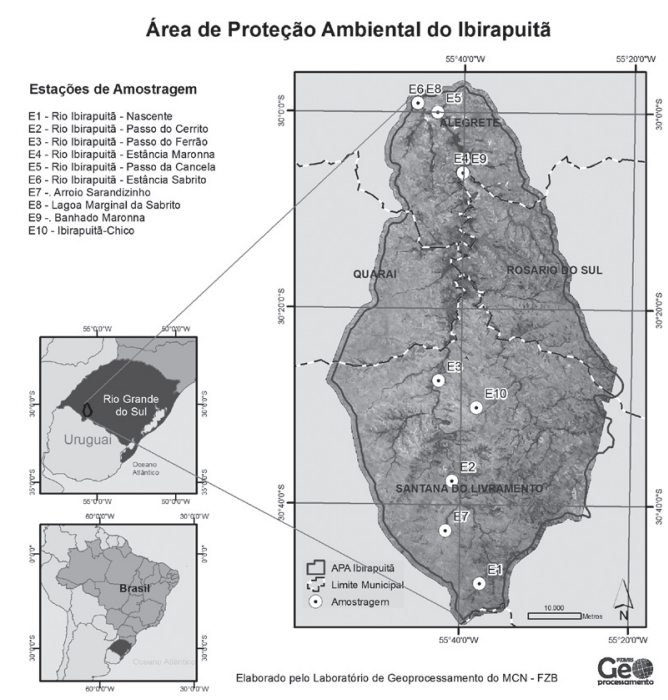

Figura 1. Localização das 10 estações de coleta (E1-E10) na Área de Proteção Ambiental do Ibirapuitã, sudoeste do Estado Rio Grande do Sul, Brasil.

Figure 1. Location of 10 sampling stations (E1-E10) in the Environmental Protection Area of Ibirapuitã, southwest of Rio Grande do Sul State, Brazil.

indispensáveis à identificação taxonômica das espécies. Totalizando 34 amostras, provenientes de quatro campanhas abrangendo duas épocas do ano (outono e primavera), no período de 2011 a 2013, em ambientes lóticos (rio e afluentes) e lênticos (lagoa marginal e banhado) localizados entre $29^{\circ} 59^{\prime} \mathrm{S}-$ $55^{\circ} 45^{\prime} \mathrm{O}$ e $30^{\circ} 48^{\prime} \mathrm{S}-55^{\circ} 37^{\prime} \mathrm{O}$.

A análise qualitativa dos 10 locais foi realizada com amostra viva e fixada entre lâmina e lamínula (média = 20), com auxílio de um microscópio Leica-DMLS com ocular-micrometrada e a imagem das espécies foi capturada com câmera digital Sony, modelo DSCW370.

A frequência de ocorrência dos táxons foi calculada segundo Mateucci \& Colma (1982) que estabeleceu as categorias: muito frequente $(\mathrm{F} \geq 70 \%)$, frequente $(40 \% \leq \mathrm{F}<70 \%)$, pouco frequente $(10 \% \leq$ $\mathrm{F}<40 \%)$ e esporádica $(\mathrm{F}<10 \%)$.
O trabalho apresenta diagnose, descrição morfométrica das células e relação entre o comprimento e largura (Rc/l) dos táxons identificados, comentários, relação do material examinado em ordem crescente de seus números de acesso no herbário, distribuição geográfica por região e Estado no Brasil, com base em trabalhos que permitem re-identificação dos táxons através de descrições, medidas, ilustrações e/ou fotomicrografias dos táxons.

O enquadramento taxonômico em nível de divisão e classe seguiu Hoeck et al. (1995) e para os níveis específico e infraespecífico dos táxons foram utilizadas obras básicas como as de Deflandre (1926), Balech (1944), Huber-Pestalozzi (1955), Starmach (1983), Tell \& Conforti (1986), Wolowski (1998) e de Shi et al. (1999); além de trabalhos como os de Couté \& Thérézian (1985), Menezes \& Fernandes (1987), Conforti (1993), Giani et al. (1999), Kim et al. (2000), Conforti \& Ruiz (2001) e Aprile \& Mera (2007).

Todas as amostras estão tombadas no Herbário Prof. Dr. Alarich R.H. Schultz (HAS) do Museu de Ciências Naturais da Fundação Zoobotânica do Rio Grande do Sul.

\section{Resultados e Discussão}

A análise resultou na identificação de nove táxons específicos e infraespecíficos de Trachelomonas na APA que são novos registros para o Estado do Rio Grande do Sul e para o Brasil.

Levantamento taxonômico

Classe Euglenophyceae

Ordem Euglenales

Família Euglenaceae

Gênero Trachelomonas Ehr. 1833 emend. Defl. 1926

Chave artificial para identificação de espécies e infraespécies de Trachelomonas.

\section{Pirenóides presentes}

2. Haplopirenóides presentes

3. Lórica com espinhos

4. Espinhos terminando em forma de lança, distribuídos em toda superfície da lórica T. hirta var. hirta

4. Espinhos terminando em forma de lança distribuídos somente nos pólos T. hirta var. duplex

3. Lórica sem espinhos

5. Lórica cilíndrica, com colarinho curto e estreito T. cylindrica var. decollata

5. Lórica com pólo anterior achatado e o posterior levemente triangular, com pontuação marcantes 
1. Pirenóides ausentes

2. Haplopirenóides ausentes

6. Lórica subesférica

7. Com colarinho levemente alargado na extremidade $4 \times 4 \mu \mathrm{m}$, parede pontuada T. wermeli

7. Sem colarinho, espessamento anelar conspícuo, pólo anterior achatado, parede lisa

T. bernardi var. major

6. Lórica amplamente elíptica ou elíptica

8. Lórica com processo caudal

9. Processo caudal mamilado, lórica amplamente elíptica, espinhos cônicos pequenos, distribuídos em toda superfície da parede

T. torosa

9. Processo caudal cônico, 2,5-4 $\mu \mathrm{m}$, lórica elíptica com lados levemente convexos em direção aos pólos T. caudata f. pseudocaudata

8. Lórica sem processo caudal

10. Lórica com espinhos cônicos, curvos, voltados em direção ao pólo posterior, espinhos posteriores $12-13 \mu \mathrm{m}$

T. spinosa

\section{Trachelomonas bernardii Woloszynska f. major} Deflandre. Nemours, p. 57, pl. 1, fig. 33, 1926.

Figuras 2-3

Lórica subesférica, 23-25 $\mu \mathrm{m}$ compr., 30-31 $\mu \mathrm{m}$ larg.; Rc/1: 0,7-0,8; pólo anterior achatado, com terço anterior mais alargado; pólo posterior arredondado; espessamento anelar conspícuo; parede lisa, castanho escura; cloroplastos numerosos, discóides, 2,5-3 $\mu \mathrm{m}$ de diâm., haplopirenóides presentes ou não.

Difere da variedade típica da espécie pelas maiores dimensões.

Material examinado: BRASIL. Rio GRANDE DO SuL: Livramento, nascente - E1, 26-III-2011, S.M. Alvesda-Silva (HAS109250); Livramento, nascente - E1, 28-III-2012, S.M. Alves-da-Silva (HAS109274); Alegrete, lagoa marginal Sabrito - E8, 27-III-2012, S.M. Alves-da-Silva (HAS109281).

Distribuição Geográfica: Primeiro registro para o Brasil.

\section{Trachelomonas caudata (Ehr.) Stein f. pseudocaudata}

(Deflandre) Popova. Flora Sporovych Rastenij SSSR. 3(1): 192, pl.18, fig. 5-6. 1966.

Figuras 4-5

Lórica elíptica, 38-42 $\mu \mathrm{m}$ compr., 17-18 $\mu \mathrm{m}$ larg., $\mathrm{Rc} / 1=2,2-2,3$; pólo anterior gradativamente atenuado em colarinho cilíndrico, ca. $5 \mu \mathrm{m}$ alt., ca. $5 \mu \mathrm{m}$ larg., bordo denteado; pólo posterior atenuado em processo caudal hialino, cônico, 2,5-4 $\mu \mathrm{m}$ compr., terminando denteado ou com três espinhos; parede levemente pontuada, castanho avermelhada, com espinhos curtos cônicos com extremidade arredondada ou não, ca. 1,5 $\mu \mathrm{m}$ compr., distribuídos irregularmente na superfície da lórica, cloroplastos numerosos, ca. $4 \mu \mathrm{m}$ de diâm.; flagelo ca. uma vez o comprimento da lórica.

Os indivíduos apresentaram características morfológicas e superposição de medidas que poderiam levar à identificação de Trachelomonas pseudocaudata ou T. caudata, razão de adotar-se a proposição da forma de Popova (1966) que considera T. pseudocaudata de Deflandre (1926) como um sinônimo homotípico de T. caudata.

Material examinado: BRASIL. Rio GRANDE do Sul: Livramento, nascente - E1, 30-XI-2011, S.M. Alvesda-Silva (HAS109261); Alegrete, lagoa marginal Sabrito - E8, 27-III-2012, S.M. Alves-da-Silva (HAS109281).

Distribuição geográfica: Primeiro registro da variedade para o Brasil.

Trachelomonas cupula Deflandre. Revue Générale de Botanique 38: 584, pl.6, figs. 97-99.1926.

Figuras 6-8

Lórica hemisférica, 8-12,5 $\mu \mathrm{m}$ compr., 7-18 $\mu \mathrm{m}$ larg.; R1/c = 0,7-1,1; pólo anterior achatado, com ângulos arredondados; espessamento anelar 2-3 $\mu \mathrm{m}$ diâm.; colarinho às vezes presente; pólo posterior levemente triangular com pontuações bem marcadas, conspícuas; parede castanho-avermelhado, cloroplastos discóides; haplopirenóides presentes.

Essa espécie assemelha-se com Trachelomonas bernardii f. major por apresentar pólo anterior achatado, distinguindo-se, entretanto, pelo pólo posterior triangular e parede com pontuações maiores e bem marcadas.

Material examinado: BRASIL. Rio GRANDE do Sul: Alegrete, lagoa marginal Sabrito - E8, 27-III-2012, S.M. Alves-da-Silva (HAS109281). 
Distribuição geográfica: Sul (Estado do Paraná). Primeiro registro para o Estado do Rio Grande do Sul.

T. cylindrica (Ehrenberg.) Playfair var. decollata Playfair. Proceedings of the Linnean Society of New South Wales 40 (1): 13, pl. 1, fig. 30. 1915

Figura 9

Lórica cilíndrica, 17-17,5 $\mu \mathrm{m}$ compr., ca. $10 \mu \mathrm{m}$ larg.; Rc/1 = 1,7; com lados paralelos; pólos arredondados, convergentes; pólo anterior com colarinho baixo e estreito; parede lisa, amarelada; cloroplastos discóides, ca. $3 \mu \mathrm{m}$ diâm.

Esta variedade diferencia-se da típica pela presença de colarinho baixo e estreito.

Material examinado: BRASIL. Rio GRANDE do Sul: Livramento, arroio Sarandizinho - E7, 1-XII-2011, S.M. Alves-da-Silva (HAS109271).
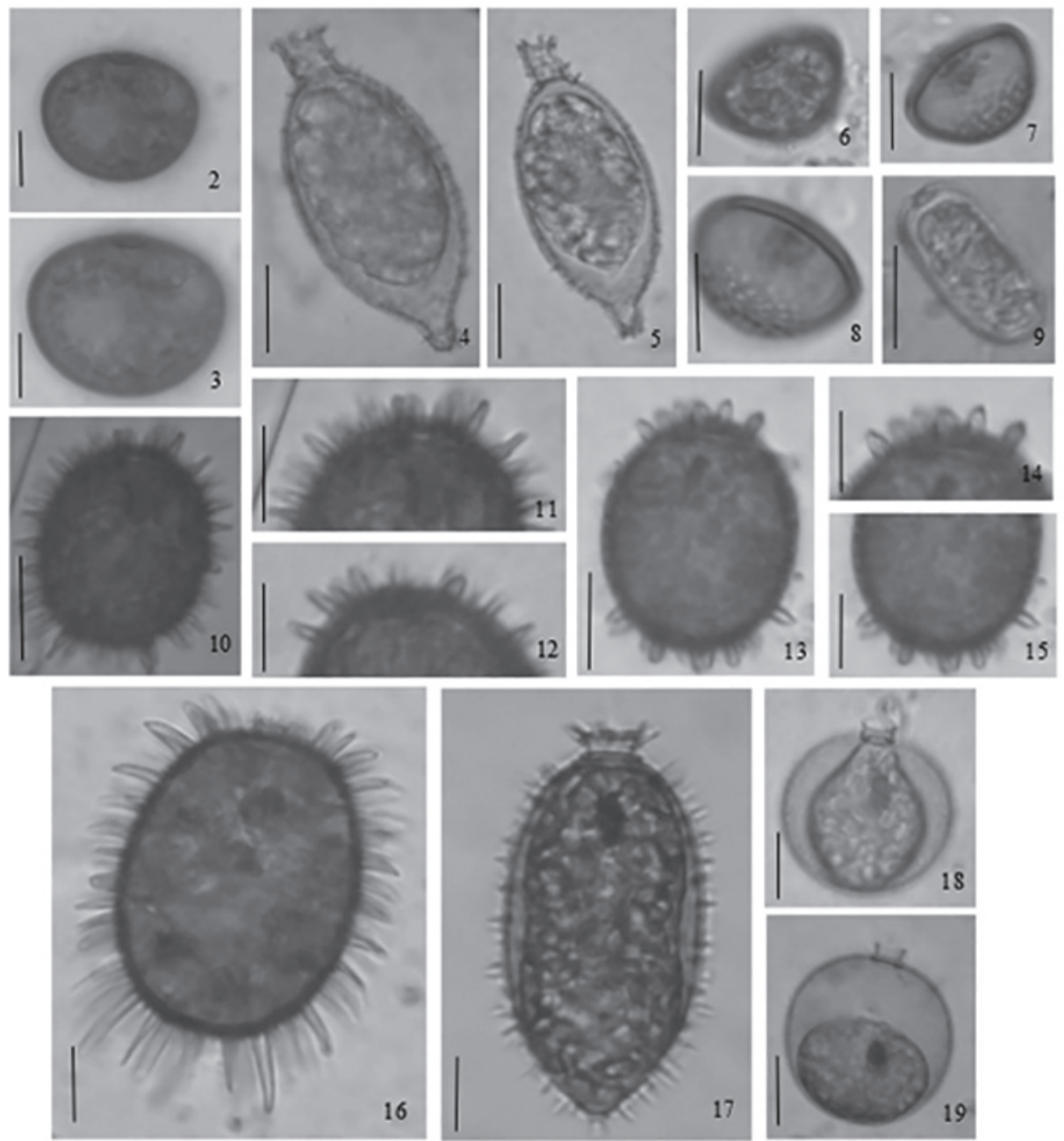

Figuras 2-19. Novos registros de Trachelomonas na APA do Ibirapuitã, RS, Brasil. 2-3. T. bernardii f. major. 4-5. T. caudata f. pseudocaudata. 6-8. T. cupula. 9. T. cylindrica var. decollata. 10-12. T. hirta var. hirta. 11-12. Detalhe dos espinhos. 13-15. T. hirta var. duplex, 14-15. Detalhe dos espinhos. 16. T. spinosa. 17. T. torosa. 18-19. Trachelomonas wermeli. Escala $=10 \mu \mathrm{m}$.

Figures 2-19. New records of the Trachelomonas in APA of Ibirapuitã, Rio Grande do Sul State, Brazil. 2. T. bernardii f. major. 4-5. T. caudata f. pseudocaudata. 6-8. T. cupula. 9. T. cylindrica var. decollata. 10-12. T. hirta var. hirta. 11-12. Detail of spines. 13-15. T. hirta var. duplex, 14-15. Detail of spines. 16. T. spinosa. 17. T. torosa. 18-19. Trachelomonas wermeli. Scale bar $=10 \mu \mathrm{m}$. 
Distribuição geográfica: Centro-Oeste (Distrito Federal, Estado de Goiás), Norte (Estados do Amazonas e Tocantins). Primeiro registro para o Estado do Rio Grande do Sul.

Trachelomonas hirta Cunha var. hirta. Memórias do Instituto Oswaldo Cruz 6: 171, pl. 24, fig. 4. 1914. Figuras 10-12

Lórica amplamente elíptica, 23-25 $\mu \mathrm{m}$ compr., 18-19 $\mu \mathrm{m}$ larg.; Rc/1 = 1,3; pólos arredondados; o anterior sem colarinho; parede castanho-avermelhada, escura, com espinhos cônicos, que afinam rapidamente no terço distal, com extremo semelhante à ponta de lança, com distribuição irregular; cloroplastos numerosos, discóides, haplopirenóides presentes.

Em microscópia óptica o terço final dos espinhos desta espécie é de difícil visualização em aumentos menores, podendo ser melhor observado em aumento de $1000 \times$. Apresenta semelhança com T. robusta Swir. emend. Defl. diferindo pelos espinhos que nessa espécie são cônicos e pelas dimensões ligeiramente maiores (Tell \& Conforti 1986).

Material examinado: BRASIL. Rio GRANDE DO SuL: Livramento, arroio Sarandizinho - E7, 24-III-2011, S.M. Alves-da-Silva (HAS109256); Livramento, nascente - E1, 30-XI-2011, S.M. Alves-da-Silva (HAS109261); Livramento, nascente- E1, 28-III-2012, S.M. Alves-da-Silva (HAS109274).

Distribuição geográfica: Primeiro registro da variedade para o Brasil.

Trachelomonas hirta Cunha var. duplex Deflandre. Bulletin de la Société Botanique de France 74: 664, fig. 6. 1928.

Figuras 13-15

Lórica elíptica, 24-29 $\mu \mathrm{m}$ compr., 19-20 $\mu \mathrm{m}$ larg.; $\mathrm{Rc} / 1=1,3-1,45$; pólos arredondados; o anterior sem colarinho; parede castanho-avermelhada, escura, com espinhos cônicos, que afinam rapidamente no terço distal, com extremo semelhante à ponta de lança, distribuídos nos dois pólos; cloroplastos numerosos, discóides, ca. $2 \mu \mathrm{m}$ diâm.; haplopirenóides presentes. Difere da variedade típica pela forma e distribuição dos espinhos somente nos pólos e pelas maiores dimensões da lórica.

Material examinado: BRASIL. Rio GRANDE Do SuL: Livramento, arroio Sarandizinho - E7, 24-III-2011, S.M. Alves-da-Silva (HAS109256); Livramento, nascente - E1, 30-XI-2011, S.M. Alves-da-Silva
(HAS109261); Alegrete, lagoa marginal Sabrito - E8, 27-III-2012, S.M. Alves-da-Silva (HAS109281).

Distribuição geográfica: Norte (Estado do Amazonas), Sudeste (Estado de São Paulo). Primeiro registro para o Estado do Rio Grande do Sul.

Trachelomonas spinosa Stokes. Proceedings of the American Philosophical Society 28:76, fig. 14. 1890

Figura 15

Lórica amplamente elíptica, 50-51 $\mu \mathrm{m}$ compr., ca. $36 \mu \mathrm{m}$ larg.; $\mathrm{Rc} / \mathrm{l}=1,4$; pólos arredondados, o anterior com colarinho baixo e largo, rodeada por 5-6 espinhos cônicos, 2-3 $\mu \mathrm{m}$ compr.; parede castanhoavermelhada, pontuada, com espinhos cônicos, curvos, voltados para o pólo posterior, distribuídos em toda extensão da lórica, os do pólo posterior, maiores, 12-13 $\mu \mathrm{m}$ compr.; 10-12 haplopirenóides presentes.

Os indivíduos apresentaram dimensões um pouco maiores que as descritas por Menezes \& Fernandes (1987) e Balech (1944). Segundo este último autor, as dimensões da espécie são 40-48 $\mu \mathrm{m}$ compr. por 30-31 $\mu \mathrm{m}$ larg. e os espinhos posteriores tem 6-12 $\mu \mathrm{m}$ compr.

Material examinado: BRASIL. Rio Grande do SuL: Livramento, nascente - E1, 30-XI-2011, S.M. Alves-da-Silva (HAS109261); Livramento, arroio Sarandizinho - E7, 1-XII-2011, S.M. Alves-da-Silva (HAS109271).

Distribuição geográfica: Centro-Oeste (Estado do Mato Grosso). Primeiro registro para o Estado do Rio Grande do Sul.

Trachelomonas torosa (Balech) Tell \& Conforti. Bibliotheca Phycologica 75: 158, fig. 368. 1986. Figura 17

Lórica elíptica a amplamente elíptica, com pólos levemente arqueados, 53-60 $\mu \mathrm{m}$ compr., 26-33 $\mu \mathrm{m}$ larg.; Rc/1 = 1,8-2,0; pólo anterior com colarinho largo e curto, ca. $2 \mu \mathrm{m}$ alt., ca. $5 \mu \mathrm{m}$ larg., com 8-10 espinhos; pólo posterior estreitando-se em um processo caudal rombudo, curto, ca. $3 \mu \mathrm{m}$; parede pontuada, castanho-avermelhada, rodeada por espinhos cônicos 2,5-3,8 $\mu \mathrm{m}$, distribuídos em toda superfície da lórica; cloroplastos, arredondados, numerosos, pequenos, ca. $2 \mu \mathrm{m}$ diâm.

Material examinado: BRASIL. Rio GRANDE do Sul: Livramento, arroio Sarandizinho - E7, 24-III-2011, S.M. Alves-da-Silva (HAS109256). 
Distribuição geográfica: Primeiro registro para o Brasil.

Trachelomonas wermeli Skvortzov. Bericht der Deutschen botanischen Gesellschaft 43:306, pl.10, fig.6. 1925

Figuras 18-19

Lórica subesférica, 28-29 $\mu \mathrm{m}$ compr., 27-28 $\mu \mathrm{m}$ larg.; Rc/1 =1,0-1,1; pólo anterior com colarinho reto, levemente alargado na extremidade, ca. $4 \mu \mathrm{m}$ alt., ca. $4 \mu \mathrm{m}$ larg.; parede pontuada, castanha clara a castanho-avermelhada clara; cloroplastos numerosos, discóides, ca. $2,8 \mu \mathrm{m}$ diâm.

Material examinado: BRASIL. Rio GRANDE Do Sul: Livramento, arroio Sarandizinho - E7, 1-XII-2011, S.M. Alves-da-Silva (HAS109271); Alegrete, lagoa marginal Sabrito - E8, 27-III-2012, S.M. Alves-daSilva (HAS109281); Alegrete, lagoa marginal Sabrito - E8, 21-V-2013, S.M. Alves-da-Silva (HAS109666).

Distribuição geográfica: Norte (Estados do Amazonas e Rondônia). Primeiro registro no Estado do Rio Grande do Sul.

Das 10 estações estudadas, foram encontradas as novas citações de Trachelomonas para o Estado e o Brasil em apenas três estações de coleta: na nascente do rio Ibirapuitã (E1), no arroio Sarandizinho (E7) e na lagoa marginal Sabrito (E8); e em somente nove amostras, ou seja, em $26,5 \%$ do total das amostras analisadas (tabela 1). Embora a nascente do rio e do arroio Sarandizinho sejam ambientes lóticos, esses apresentaram visualmente baixíssima correnteza, e profundidade média entre $0,36-0,40 \mathrm{~m}$ devido à longa estiagem verificada no período de 2011 a 2012, o que deve ter propiciado a presença dos táxons nesses ambientes.

A maior riqueza foi registrada na lagoa marginal Sabrito com a ocorrência de T. bernardii f. major, $T$. caudata, T. cupula, T. hirta var. duplex e T. wermeli. Nessa unidade amostral, no outono (27/03) de 2012, ocorreu grande diversidade de Euglenophyceae e foram observados cerca de 250 indivíduos por lâmina de T. wermeli.

Foram espécies exclusivas da lagoa marginal Sabrito, T. cupula e do arroio Sarandizinho, T. cylindrica var. decollata. e T. torosa.

Todos os nove táxons foram esporádicos na APA e com baixa distribuição no Brasil, com exceção de T. cylindrica var. decollata já registrada em quatro Estados.

O estudo permitiu a ampliação do conhecimento da distribuição geográfica do gênero no Estado do Rio Grande do Sul e Brasil. São registrados pela primeira vez no país $T$. bernardii f. major, $T$. caudata var. pseudocaudata, T. hirta var. hirta e T. torosa, enquanto os demais táxons são primeira citação para o Estado.

Tabela 1. Distribuição dos novos registros de Trachelomonas na APA do Ibirapuitã, Estado do Rio Grande do Sul, Brasil, nas três estações de coleta, entre 2011 a 2013. E1: nascente do Rio Ibirapuitã, E7: arroio Sarandizinho, E8: lagoa marginal da Sabrito.

Table 1. Distribution of new records of the Trachelomonas in APA of Ibirapuitã, Rio Grande do Sul State, Brazil, in three sampling stations between 2011 and 2013. E1: Source of the Ibirapuitã River, E7: Sarandizinho Stream, E8: marginal pond of the Sabrito.

\begin{tabular}{|c|c|c|c|c|c|c|c|c|c|}
\hline Estações de coleta / Datas & Est. 1 & Est. 1 & Est. 1 & Est. 7 & Est. 7 & Est. 8 & Est. 8 & Est. 8 & Est. 8 \\
\hline Táxons de Trachelomonas & $26 / 3 / 11$ & $30 / 11 / 11$ & $28 / 3 / 12$ & $24 / 3 / 11$ & $1 / 12 / 11$ & $22 / 3 / 11$ & $29 / 11 / 11$ & $27 / 3 / 12$ & $21 / 5 / 13$ \\
\hline T. bernardiif. major & $\mathrm{X}$ & & $\mathrm{X}$ & & & & & $\mathrm{X}$ & \\
\hline T. caudata f. pseudocaudata & $\mathrm{X}$ & & & & & & $\mathrm{X}$ & $\mathrm{X}$ & \\
\hline T. cupula & & & & & & & & $\mathrm{X}$ & \\
\hline T. cylindrica var. decollata & & & & & $\mathrm{X}$ & & & & \\
\hline T. hirta var. hirta & & $\mathrm{X}$ & $\mathrm{X}$ & $\mathrm{X}$ & & & & & \\
\hline T. hirta var. duplex & & $\mathrm{X}$ & & $\mathrm{X}$ & & & & $\mathrm{X}$ & \\
\hline T. spinosa & & $\mathrm{X}$ & & & $\mathrm{X}$ & & & & \\
\hline T. torosa & & & & $\mathrm{X}$ & & & & & \\
\hline T. wermeli & & & & & $\mathrm{X}$ & & & $\mathrm{X}$ & $\mathrm{X}$ \\
\hline
\end{tabular}




\section{Agradecimentos}

À Fundação de Amparo à Pesquisa do Estado do Rio Grande do Sul, pela Bolsa de Iniciação Científica (PROBIC) à coautora Karine Corrêa Escobar (Proc. 0430-2551/12-6). À Chefe da APA do Ibirapuitã/ICMBio-RS Eridiane Lopes da Silva e ao Raul Paixão Coelho, pelo apoio e disponibilidade durante as campanhas realizadas. Aos colegas da Seção de Botânica de Criptógamas, Zulanira Meyer Rosa, Vera Regina Werner e Manoel Luiz Nunes e da Seção de Invertebrados aquáticos do Museu de Ciências Naturais da Fundação Zoobotânica do Rio Grande do Sul, Ana Luiza Miranda, pelo auxílio e companheirismo nas expedições de coleta; ao Tomaz Aguzzoli e Mariano C. Pairet Junior, pelo apoio em campo; ao Ricardo Aranha e Mauricio de Freitas Scherer da Seção de Geoprocessamento, pelo georeferenciamento dos locais e mapa da área.

\section{Literatura citada}

Alves-da-Silva, S.M. 1988. Euglenaceae pigmentadas (Euglenophyta) da Estação Ecológica do Taim, Rio Grande do Sul, Brasil, Iheringia, série Botânica 38: 109-126.

Alves-da-Silva, S.M. \& Ávila, I.R. 1995. O gênero Trachelomonas Ehr. emend. Defl. (Euglenaceae) do Parque Zoológico da Sapucaia do Sul e do Jardim Botânico de Porto Alegre, Rio Grande do Sul, Brasil. Iheringia, série Botânica 46: 27-56.

Alves-da-Silva, S.M. \& Bicudo, C.E.M. 2013. Taxonomic and ultrastructural survey of Trachelomonas (Euglenophyceae) of a shallow subtropical reservoir in the municipality of Triunfo, southern Brazil. Brazilian Journal of Botany 36: 223-246.

Alves-da-Silva, S.M. \& Laitano, C.S. 1994. Euglenaceae Pigmentadas do Banhado do Jacaré, em um Parque de Proteção Ambiental, Triunfo, Rio Grande do Sul, Brasil. Iheringia, série Botânica 45: 89-116.

Alves-da-Silva, S.M. \& Klein, I.C. 2015. Euglenophyceae na Área de Proteção Ambiental do Rio Ibirapuitã, sudoeste do Estado do Rio Grande do Sul, Brasil. 1. Cryptoglena Marin \& Melkonian emend. Kosmala \& Zakrýs, Monomorphina (Ehrenberg) Mereschkowsky emend. Kosmala \& Zackýs e Phacus Durjardin. Hoehnea 42: 471-496.

Alves-da-Silva, S.M. \& Schüler-da-Silva, A. 2007. Novos registros do gênero Trachelomonas Ehr. (Euglenophyceae) no Parque Estadual Delta do Jacuí e no Rio Grande do Sul, Brasil. Acta Botanica Brasilica 21: 401-409.

Alves-da-Silva, S.M. \& Tamanaha, M.S. 2008. Ocorrência de Euglenophyceae pigmentadas em rizipiscicultura na Região do Vale do Itajaí, SC, Sul do Brasil. Acta Botanica Brasilica 22: 145-163.
Alves-da-Silva, S.M., Hermany, G. \& Oliveira, M.A. 2007. Diversity and ecological considerations on pigmented Euglenophyceae in the State Park of The Jacuí Delta, Rio Grande do Sul, Southern Brazil. Biociências 15: 8-20.

Alves-da-Silva, S.M., Juliano, V. \& Ferraz, G.C. 2008. Euglenophyceae pigmentadas em lagoa ácida rasa, Parque Estadual de Itapuã, sul do Brasil. Iheringia, série Botânica 63: 15-36.

Alves-da-Silva, S.M., Cabreira J. da C., Voss, J.G. \& Lobo, E.A. 2013. Species richness of the genera Trachelomonas and Strombomonas (pigmented Euglenophyceae) in a subtropical urban lake in the Porto Alegre Botanical Garden, RS, Brazil. Acta Botanica Brasilica 27: 526-536.

Aprile, F.M. \& Mera, P.A.S. 2007. Fitoplâncton e fitoperifíton de um rio de águas pretas da Amazônia periférica do Norte, Brasil. Brazilian Journal of Aquatic Science and Technology 11: 1-14.

Araújo, G.J.M., Barbosa, J.E.L. \& Barbosa, L.G. 2012. Pigmented Euglenophytes in a natural and shallow lake in the semiarid region of Paraíba State, Brazil. Brazilian Journal of Botany 35: 17-30.

Balech, E. 1944. Trachelomonas de la Argentina. Anales del Museo Argentino de Ciencias naturales Bernardino Rivadavia 41: 223-305.

Bicudo, C. E.M. \& Menezes, M. 2006. Gênero de algas de águas continentais do Brasil (chave para identificação e descrições). 2 ed. Rima, São Carlos.

Bourrelly, P.C. 1970. Les algues d'eau douce: initation à la systematique, III. Les algues blues et rouges, les Eugléniens, Peridiniens et Cryptomonadines. Éditions N. Boubée, Paris.

Cardoso, M.B. 1982. Levantamento das Euglenaceae pigmentadas do Distrito Federal, Brasil. Tese de Doutorado, Universidade de São Paulo, São Paulo.

Conforti, V.T.D. 1993. Study of the Euglenophyta from Camaleão lake (Manaus-Brazil). I. Trachelomonas. Revue d'Hydrobiologie Tropicale 26: 3-18.

Conforti, V. \& Tell, G. 1986. Ultraestructure de la loriga de Trachelomonas Defl. (Euglenophyta) em microscópio electronico de barrido (M.E.B). Nova Hedwigia 43: 45-79.

Conforti, V.T.D. \& Ruiz, L. 2001. Euglenophytes from Chuman Reservoir (South Korea) II Trachelomonas Ehr. Algological Studies 102: 117-145.

Conforti, V., Lionardi, M., Segura, M. \& Rojo, C. 2005. Las Euglenofítas en las Tablas de Daimiel como ejemplo de las limitaciones de los indicadores biológicos de la degradación ambiental. Anales del Jardín Botánico de Madri 62: 163-179.

Conrad, W. 1916. Revision des espèces indigènes et françaises du genre Trachelomonas Ehrenberg. Annales de biologie lacustre 8: 193-212. 
Conti, J.B. \& Furlan, S.A. 2003. Geoecologia: o clima, os solos e a biota. In: J.L. Ross (org.). Geografia do Brasil. Editora da Universidade de São Paulo, São Paulo, pp. 67-2017.

Couté, A. \& Thérézian, Y. 1985. Primière contribution à l'étude des Trachelomonas (Algae, Euglenophyta) de l'Amazonie bolivienne. Revue d'Hydrobiologie Tropicale 18:111-131.

Cunha, A.M. 1913. Contribuição para o conhecimento da fauna de protozoários do Brasil. Memórias do Instituto Oswaldo Cruz 5: 101-122.

Cunha, A.M. 1914. Contribuição para o conhecimento da fauna de protozoários do Brasil. Memórias do Instituto Oswaldo Cruz 6: 169-179.

Danilov, R.A. \& Elelund, G.A. 2001. Phytoplankton communities at different depths in two eutrophic and two oligotrophic temperate lakes at higher latitude during the period of ice cover. Acta Protozoologica 40: 197-201.

Deflandre, G. 1926. Monographie du genre Trachelomonas Ehrenberg. Nemours, André Lesot (cópia xerografada).

Domingues, C.D. \& Torgan, L.C. 2011. Fitoplâncton (exceto Chlorophyceae) de um lago artificial urbano, sul do Brasil. Revista Brasileira de Botânica 34: 463-480.

Duangjan, K., Wolowski, K. \& Peerapornpisal, Y. 2012. A taxonomic and Ultrastrucutral study of Trachelomonas spp. (Euglenophyta) from Agricultural Area Pond, Lamphun Provice. Journal of the Microscopy Society of Thailand 5: 23-27.

Ehrenberg, C.G. 1833. Dieter Beiträg zur Erkenntnis grosser Organisation in der Richtung des Kleinstein Raumes. Physikalische Abhandlungen der Akademie der Wissinschaften, Berlin, pp. 145-336.

Ferreira, A.C. \& Menezes, M. 2000. Flora planctônica de um reservatório eutrófico, Lagoa Guandu, município de Nova Iguaçu, RJ. Hoehnea 27: 45-76.

Franceschini, I.M. 1992. Algues d'eau douce de Porto Alegre Brésil (les Diatomophycée exclues). Biblioteca Phycologica 92: 1-81.

Giani, A., Figueredo, C. \& Eterovick, P. 1999. Algas planctônicas do reservatório da Pampulha (MG): Euglenophyta, Chrysophyta, Pyrrophyta, Cyanobacteria. Revista Brasileira de Botânica 22: 107-116.

Guiry, M.D. \& Guiry, G.M. 2014. AlgaeBase. World-wide electronic publication, National University of Ireland, Galway. Disponível em http://www.algaebase.org (acesso em 18-V-2014).

Hoek, C. van den, Mann, D.G. \& Jahns, H.M. 1995. Algae: An introduction to phycology. Cambrige University Press, Cambridge.

Huber-Pestalozzi, G. 1955. Euglenophyceen. In: G. HuberPestalozzi (ed.). Das phytoplankton des Susswässers: Systematik und Biologie. E. Schweizerbart'sche Verlangsbuchhandlug, Stuttgart 16: 1-605.
Jati, S. \& Train, S. 1994. Euglenaceae pigmentadas de ambientes lênticos da Ilha Porto Rico, município de Porto Rico, Paraná, Brasil. Iheringia, série Botânica 45: 117-142.

Keppeler, E.C., Lopes, M.R.M. \& Lima, C.S. 2002. Ficoflórula do lago Amapá em Rio Branco - Acre, I: Euglenophyceae. Revista Brasileira de Biologia 59: 679-686.

Kim, J.T. \& Boo, S.M. 2001. The relationships of green euglenoids to environmental variables in Jeonjucheon, Korea. Korean Journal of Limnology 34: 81-89.

Kim, J.T., Boo, S.M. \& Couté, A. 2000. Taxonomic and floristic accounts of the genus Trachelomonas Ehrenberg, 1933 (Euglenophyceae) from Korea. Korean Journal of Limnology 33: 80-108.

Lemmermann, E. 1913. Eugleninae. In: Pascher, A. Die Süsswasserflora Deutschlandes Österreichs und der Schweiz, Jena: Gustav Fischer Verlag., v. 2, pp. 115-174.

Matsubara, C.P., Maltchik, L. \& Torgan, L.C. 2008. Diversidade e distribuição de algas em áreas úmidas do Rio Grande do Sul, Brasil. Neotropical Biology and Conservation 3: 21-27.

Menezes, M. 1991. Trachelomonas (Euglenophyceae) do município do Rio de Janeiro e arredores, Rio de Janeiro, Brasil. Hoehnea 18: 57-73.

Menezes, M. \& Fernandes, V.O. 1987. Euglenaceae pigmentadas do município de Cáceres e arredores, Mato Grosso, Brasil: uma contribuição ao seu conhecimento. Rickia 14: 53-71.

Menezes, M., Nascimento, E.P. \& Fonseca, C.G. 1995. Criptógamos, Euglenophyceae. In: I.F. Campos (org.). Flora dos Estados de Goiás e Tocantins. Universidade Federal de Goiás, Goiânia, v. 4, n.1, pp. 1-77.

Middelhoek, A. 1948. À propos de quelques espèces du genre Trachelomonas Ehrenb. et du genre Strombomonas Defl. trouvées aux Pays-Bas. I. Hydrobiologia 1: 78-79.

Middelhoek, A. 1951. A propos de quelques espéces du genre Trachelomonas Ehrbg. et du genre Strombomonas Defl. trouvées aux Pays-Bas. III. Hydrobiologia 3: 228-243.

Pérez, M.D.C. 2002. Fitoplancton del rio Negro, Uruguai. Limnetica 21: 81-92.

Philipose, M.T. 1988. Contribution to our knowledge of Indian algae 3. Euglenineae Part 3. The genera Trachelomonas Ehrenberg and Strombomonas Deflandre. Proceedings Indian Academy Sciences 98: 317-394.

Playfair, G.I. 1915. The genus Trachelomonas. Procedings of the Linnean Society of New South Wales 40: 1-41.

Popova, T.G. 1966. Evglenovyje vodoroski vyp. 1. Flora sporovych rastenijj Izd. Nauka, Moscou.

Pringshein, E.G. 1953. Observations on some species of Trachemononas grown in culture. New Phytologist 53: 93-113. 
Pringsheim, E.G. 1956. Contributions towards a monograph of the genus Euglena. Nova Acta Leopoldina 18: 1-168.

Relatório Brasil das Águas. Sete Rios. Rio Ibicuí. 2007. Gerald \& Margi Moss. Disponível em http://www. brasildasaguas.com.br (acesso em 30-X-2009).

Rino, J.A. 1972. Contribuição para o conhecimento de algas de água doce de Moçambique III. Revista de Ciências Biológicas, Sér. A, 5: 149-172.

Rino, J.A. \& Pereira, M.J. 1988. Euglenophyta da região centro de Portugal I. Gênero Trachelomonas Ehr. 1833 emend. Defl. 1926. Revista de Biologia da Universidade de Aveiro 2: 129-161.

Rino, J.A. \& Pereira, M.J. 1989-1990. Euglenophyta da região centro de Portugal. II. Gênero Trachelomonas Ehr. 1833 emend. Defl. 1926. Estrutura da lorica em microscopia electronica de varrimento. Revista de Biologia da Universidade de Aveiro 3: 139-187.

Rodrigues, L.L.R. 2008. Biodiversidade de cianobactérias e algumas das Represas Billing (Braço Taquarecetuba) e Guarapiranga, SP, Brasil. Dissertação de Mestrado, Universidade de São Paulo, São Paulo.

Rosa, Z.M., Ungareti, I., Kremer, L.M., Alves-da-Silva, S.M., Callegaro, V.L., Werner, V.R. 1987. Ficoflora de ambientes lênticos - estudo preliminar da região de Charqueadas, Rio Grande do Sul, Brasil, com vistas à avaliação ambiental. Acta Botanica Brasilica 1: 165-188.

Shi, Z., Wang, Q., Xie, S., Dai, J. \& Chen, L. 1999. Euglenophyta. (s.l.). Flora Algarum Sinicarum Aquae Dulcis, t. 6, Science Press, Beijing.
Sládecék, V. 1973. System of water quality from the Biological Point of View. Archiv für Hydrobiologie 7: $1-218$

Solórzano, G.G., Martinez, M.G. O. Garfias, M.B.M. \& Conforti, V. 2011. Trachelomonas (Euglenophyta) from a eutrophic reservoir in Central Mexico. Journal of Environmental Biology 32: 463-471.

Starmach, K. 1983. Euglenophyta. In: K. Starmach (ed.). Flora Slodkowodna Polski, v. 3. Polska Academia Nauk, Warszawa.

Stein, F.R. 1878. Der organismus der Infusionsthiere, III. Der Organismus der Flagellaten I. Leipzig.

Tell, G. \& Conforti, V.T.D. 1986. Euglenophyta pigmentadas de la Argentina. Biblioteca Phycologica 75: 1-301.

Tucci, A., Sant'Anna, C.L., Gentil, R.C. \& Azevedo, M.T. de P. 2006. Fitoplâncton do Lago das Garças, São Paulo, Brasil: um reservatório urbano eutrófico. Hoehnea 33: 147-175.

Xavier, M.B. 1993. Distribuição vertical das Euglenaceae pigmentadas do Rio Grande, Represa Billings, São Paulo, Brasil. Acta Limnologica Brasiliensia 6: 11-30.

Xavier, M. B. 1994. Criptógamos do Parque Estadual das fontes do Ipiranga, São Paulo, SP. Algas, 5: Euglenaceae pigmentadas). Hoehnea 2: 47-73.

Xavier, M.B. 1996. Fitoplâncton do Rio Grande, Represa Bilings, São Paulo, Brasil: estudo taxonômico (19851996). Iheringia, série Botânica 47: 103-122.

Wolowski, K. 1998. Taxonomic and environmental studies on euglenophytes of the Kraków-Czestochowa Upland (Southern Poland). Fragmenta Floristica et Geobotanica 6: 1-192. 